\title{
DEVELOPMENT OF A STRUCTURED VIRTUAL REALITY CURRICULUM FOR LAPAROSCOPIC APPENDICECTOMY
}

Daniel M. Sinitsky ${ }^{1}$, Bimbi Fernando ${ }^{1}$, Henry Potts ${ }^{2}$, Panagis Lykoudis ${ }^{1}$, George Hamilton $^{1}$, Pasquale Berlingieri ${ }^{1,3,4}$

1. Division of Surgery \& Interventional Science, Royal Free Campus, UCL, London, UK

2. Institute of Health Informatics, UCL, London, UK

3. Centre for Screen-Based Medical Simulation, Royal Free Hospital, London, UK

4. To whom correspondence should be addressed:

Centre for Screen-Based Medical Simulation

Royal Free Hospital, London, NW3 2QG, UK

Email: p.berlingieri@ucl.ac.uk

Tel: 02077940500 xtn 36857

Word count: 3,312 (excluding abstract, references, tables, figures and legends) 


\section{ABSTRACT}

Background: Laparoscopic appendicectomy (LA) is a common surgical emergency procedure mainly performed by trainees. The aim was to develop a step-wise structured virtual reality (VR) curriculum for LA to allow junior surgeons to hone their skills in a safe and controlled environment.

Methods: A prospective randomized study was designed using a high-fidelity VR simulator. Thirty-five novices and 25 experts participated in the assessment and their performances were compared to assess construct validity. Learning curve analysis was performed.

Results: Five of the psychomotor tasks and all appendicectomy tasks showed construct validity. Learning was demonstrated in the majority of construct-valid tasks. A novel goaldirected VR curriculum for LA was constructed.

Conclusions: A step-wise structured VR curriculum for LA is proposed with a framework which includes computer generated metrics and supports deliberate practice, spacing intervals, human instruction/feedback and assessment. Future study should test the feasibility of its implementation and transferability of acquired skill.

\section{KEYWORDS}

Curriculum development; Laparoscopic appendicectomy; Simulation; Surgical training; Virtual reality. 


\section{INTRODUCTION}

Acute appendicitis is the most common abdominal surgical emergency [1], with an annual incidence in the US and the UK of 250,000 and 50,000 patients respectively [2]. One of the current standards of treatment is surgical excision, appendicectomy, with its laparoscopic management being widely accepted and routinely performed because of the associated considerable advantages: significant reduction in the incidence of wound infections, hospital stay and postoperative complications [3].

Laparoscopic surgery requires unique technical skills that are not transferable from open surgery [4]. These should be ideally acquired in a safe, controlled and standardized environment since the early part of the learning curve is associated with an increased incidence of complications [5-6]. The use of Virtual Reality (VR) laparoscopic simulation is well known; it enables measurement of performance metrics and the construction of elaborate scenes for exercises that can be repeated at a moment's notice. A systematic review from Larsen and colleagues concluded that VR laparoscopic training improves performance and reduces errors in both animal and human studies, suggesting its greatest impact has been with modules incorporating actual procedures rather than basic exercises [7].

Over the last ten years, surgical educators have progressively focussed their attention on the development of structured training curricula with the aim of providing trainees with the relevant expertise needed for their surgical practice [8-11]. Since the majority of laparoscopic appendectomies are performed by surgeons in training [12], it is imperative that they are equipped with the necessary skills early in their career. One such procedural module is the laparoscopic appendicectomy, available on the LAP Mentor (Simbionix Corporation, Cleveland, Ohio, USA), a high-fidelity VR simulator. It essential therefore to accurately investigate and assess construct validity (CV) of the LAP Mentor appendicectomy module before its incorporation into a training programme. The aim of this study was to develop a step-wise structured VR curriculum for laparoscopic appendicectomy with demonstrated construct validity. 


\section{MATERIAL AND METHODS}

\section{Setting \& Simulator}

The study was conducted at the Centre for Screen-Based Simulation of the Royal Free Hospital, a 24-hour open-access simulation center, home to eight VR simulators. The LAP Mentor II is a high-fidelity VR surgical simulator with haptic feedback which is equipped with several surgical training modules. For the purpose of this investigation only the basic psychomotor skills and the appendicectomy modules were used. In the psychomotor skills module, Task 1 - Camera Manipulation at $0^{\circ}$ (PST1) is an exercise requiring navigation using a 0-degree camera, Task 2 - Camera Manipulation at 30 (PST2) is similar but using a 30-degree camera, Task 3 - Eye-Hand Coordination (PST3) requires the participant to touch colored balls at varying depths that specify the hand that must be used, Task 4 - Clip Applying (PST4) involves applying clips with one hand to a leaking vessel, Task 5 - Clipping and Grasping (PST5) appears similarly but requires grasping with one hand and clipping with the other, Task 6 Two Handed Manoeuvres (PST6) requires manipulation of an object to allow translocation of simulated marbles with the opposite hand, Task 7 - Cutting (PST7) requires retraction with one hand and cutting of a circle with the other, Task 8 - Electrocautery (PST8) is an exercise using hook diathermy against an array of bands, and finally Task 9 - Translocation of Objects (PST9) requires manipulation of objects in order to orientate them correctly.

The laparoscopic appendicectomy module consists of two parts. In the first, there are five separate "procedural" tasks (Figure 1) that guide the participant using color codes and arrows (LAPT1 to LAPT5). LAPT1 is an exercise that requires retraction of the appendix with one instrument, and dissection of a mesenteric window with the other. LAPT2 is similar, but also requires the division of the appendicular artery following the application of three clips. LAPT3 is similar to LAPT2 though also requiring the division of the appendix using scissors, following the application of three ligating loops to specified areas at its base. LAPT4 involves division of the mesoappendix and appendix base using a stapling device. LAPT5 is similar to LAPT2, but instead requires application of an energy device to divide the artery safely. Part two of the appendicectomy module is the full appendicectomy procedure (LAFP) without color coding or prompts, ending after the detached appendix has been extracted in an endobag removal device. 
Upon completion of each task, the simulator displays the results for all of the performance metrics. There are both general- and task-specific metrics, and they can be performance- or error-related. Number of movements and path length for each hand were manually combined to yield total number of movements and total path length, respectively.

\section{Participants}

Participants were invited and assigned according to their level of laparoscopic experience into two groups: novices and experts. The novice group included foundation year one and two (FY1 and FY2) doctors who were recruited from the North Central and East Thames London Foundation Postgraduate Training Schools; they are undertaking a two-year general postgraduate clinical training programme before entering specialist training. For their inclusion, each novice participant must have been primary operator for <10 laparoscopic procedures (excluding diagnostic laparoscopy). The group of experts consisted of senior registrars and consultants who were recruited from a range of hospitals within University College London Partners (UCLP). For their inclusion, experts must have been primary operator for $\geq 100$ laparoscopic procedures (excluding diagnostic laparoscopy) and in current regular practice. Both novices and experts with prior experience on the LAP Mentor were excluded.

\section{Randomisation}

Novices $(N)$ and experts $(E)$ were each randomized by the 'closed envelope' technique to one of three groups: psychomotor skills (N1 and E1), appendicectomy procedural tasks (N2 or E2) or the full appendicectomy procedure (N3 or E3).

\section{Study Protocol}

All participants completed a pre-course questionnaire to gather information with respect to previous experience in real and simulated laparoscopy; in addition, data on sport, instruments and video games were also collected. Participants then signed a form indicating their consent to participate in the study and their agreement to adhere to the study protocol. Each participant received a standardized 15-minute one-to-one familiarization session on the simulator, including combined observation with limited participation in each of the tasks relevant to their group. Standardized techniques that were previously agreed by the authors were demonstrated for each task, and all participants were instructed to strictly adhere to 
these. Novices then returned on a separate day to commence training, while experts commenced on the same day as their familiarization session.

During training, novices were required to complete 10 sessions, each comprising one complete performance of all the tasks required of their group in consecutive order. Participants in the LAFP were stipulated to perform the procedure by dissecting the mesoappendix, double-clipping the appendicular artery before dividing it, and dividing the appendix base following the application of three ligating loops. For all participants, no more than two sessions could be performed on the same day, and no two sessions within one hour of each other. Experts completed their training in a similar manner. However, they were required to complete only two sessions, both occurring on the same day. After each performance, all participants were able to view their metric data.

In order to guarantee consistency, all participants were individually supervised by the same author (PB). This was to ensure strict adherence to the study protocol and to provide technical support with the simulator if needed. Instruction and feedback were prohibited.

\section{Power analysis}

Sample size was calculated based on a two-tail test, where $\alpha=0.05$ and power $(1-\beta)=0.80$, with a minimum expected reduction in task completion time in each expert sub-group compared to the corresponding novice sub-group of $30 \%$ based on previous studies $[13,14$, $15,16]$. This yields eight participants per sub-group. Allowing for drop-outs, the sample size was set at 36 novice and 27 expert participants.

\section{Outcome Measures \& Statistical analysis}

Performance data were anonymized and collated into a Microsoft Excel 2010 version 14.0 spreadsheet (Microsoft, Redmond, WA, USA) and stored on a secure University College London computer. Data was then transferred to IBM SPSS ${ }^{\circledR}$ version 25.0 (Armonk, NY, USA) for statistical analysis.

Data distribution for each metric across all simulator tasks was assessed using the ShapiroWilk test. For metrics where data was normally distributed, CV was assessed by comparing 
the mean of the first two repetitions in the novice group with that of the corresponding expert group using a two-tailed t-test. The two-tailed Mann-Whitney $U$ test was used to compare medians and the Fischer's exact $\chi^{2}$ test was used to compare error scores. The presence of a learning curve among novices was assessed for each construct-valid metric using the repeated measures ANOVA test for parametric data, and the Friedman test for non-parametric data.

Results were considered significant where $p<0.05$. A metric was deemed construct-valid if performances were significantly different between experts and novices. A task was deemed construct-valid if it contained one or more construct-valid metric.

\section{Curriculum Design}

The VR Laparoscopic Appendicectomy Curriculum was designed using the methodology adopted by Aggarwal et al [8]. The curriculum also heeded recommendations previously published elsewhere by the current authors [9]. Criteria for the design of the curriculum are outlined in Table 1.

\section{Ethical Approval}

Formal ethical approval was not required because no patients were enrolled in the study. This investigation involved purely the use of educational tests, its information recorded in such manner that participants cannot be identified.

\section{RESULTS}

\section{Participants and Data}

Thirty-six novices were randomized to groups N1 to N3 although one participant dropped out between induction and commencement of data collection, leaving groups N1 and N2 each with 12 participants and N3 with 11. One further participant in group N2 dropped out after the $6^{\text {th }}$ repetition (data from repetitions one to six was included in the analysis), while the remaining 34 participants attempted all 10 repetitions of their allocated task(s). Reasons for dropping out were not provided. The baseline characteristics of groups N1, N2 and N3 are presented in Table 2. 
Twenty-seven experts were recruited, with two dropping out between study induction and commencement of the first session (one citing a busy schedule, the other not providing a reason), leaving groups E1 and E2 each with eight participants, and group E3 with nine. All of the 25 remaining expert participants attempted two repetitions of their allocated task(s).

Unexplained software failures led to missing data affecting a number of movement metrics in PST4 and PST6. Missing data from either or both of a participant's first two repetitions led to the exclusion of participants from the CV analysis of these metrics (one to four novices and zero to six experts, depending on the metric).

\section{Construct Validity \& Learning Curves}

Tasks 3, 5, 6, 8 and 9 of the psychomotor skills module demonstrated CV. Thirteen of 19 (68\%) metrics demonstrated $\mathrm{CV}$ for task 5, with zero to five metrics demonstrating $\mathrm{CV}$ for all other psychomotor skills tasks. Participants demonstrated learning in 20 of these 23 (87\%) construct-valid metrics.

All five appendicectomy procedural tasks were construct-valid, with 41 of all 58 (71\%) metrics in this group demonstrating CV and all but four of these (90\%) demonstrated learning. In the full appendicectomy procedure, nine of 12 (75\%) metrics demonstrated CV, with all of these construct-valid metrics demonstrating learning. Injury to the appendicular artery demonstrated CV only in LAPT3, although significant learning was not demonstrated. CV results for the five appendicectomy procedural tasks (LAPT1-5) and for the full appendicectomy procedure (LAFP) are presented in Table 3 and Table 4 respectively, while total time learning curves for appendicectomy tasks LAPT1-5 and LAFP are presented in Figure 2.

\section{The Curriculum}

The VR Laparoscopic Appendicectomy Curriculum is illustrated in Figure 3. 


\section{DISCUSSION}

This study is a prospective randomized assessment of the LAP Mentor appendicectomy module, with all five procedural tasks and the full procedure able to differentiate between experienced surgeons and novices, together with several of the basic psychomotor skills tasks. Learning curves were also significant for the majority of construct-valid metrics, which is important for demonstrating that performance can improve towards expert proficiency by practicing on the simulator. This informed the construction of a VR laparoscopic appendicectomy curriculum.

In the present study, findings of CV in the psychomotor skills tasks partially corroborate those of others [8, 19-20], with some notable exceptions. Aggarwal et al's CV results for task 5 [8] were limited to total time and total speed, whereas in the current analysis the majority of task 5 metrics demonstrated CV. Aggarwal and colleagues also demonstrated CV for tasks 1 and 2, though this was not proved in the present study, nor by McDougall et al [20] where there was CV for tasks 3 to 9 only. Conflicting results are exemplified in the analysis for task 3 alone, where Yamaguchi et al [13], Wilson et al [16], and the present study disagree on metrics such as number of movement (left), average speed (left), relevant path length (right), and total path length (right). Such inconsistencies in the literature may be related to different studies' methodologies, with significant variations in laparoscopic experience between novice groups, and in the repetitions used for statistical analysis [21].

Unfortunately, system errors resulted in the loss of some data points for psychomotor tasks 4 and 6 in the present study, which may have resulted in underpowered analysis of some movement metrics, although it is notable that all but one metric (ideal path length, left), did not demonstrate CV. Besides this, the present study was strengthened by a unique fullysupervised training regimen that ensured strict adherence to a protocol where instruction and feedback were prohibited, guaranteeing data integrity. Another robust feature is the randomized, prospective study design that aimed to reduce the warm-up effect on later tasks in novices and experts by separating them both into task- or module-specific groups. 
To date, CV has been demonstrated in procedural modules across a variety of VR simulators, such as the MIST nephrectomy [22], LapSim salpingectomy [23], LAP Mentor cholecystectomy [8] and sigmoid colectomy modules [11]. The present LAP Mentor appendicectomy module curriculum is based on a model of deliberate practice - where learners reflect and consciously seek improvement of performance with each repetition, to achieve expert performance rather than arrested development [24]. This is accomplished by a) including tasks that have been deliberately selected to improve skill [25], b) providing specific goals, which accelerates learning during VR training $[7,20], c)$ providing immediate feedback (performance metrics), and d) being self-directed and tailored, allowing trainees to rehearse in their own time, and directing them to repeat and concentrate on tasks that are not yet expertly performed.

Core psychomotor skills tasks were included in the curriculum to create a gradual, step-wise increase in task complexity, which was deemed an appropriate strategy aimed at surgical novices. Tasks and metrics that demonstrated CV were included with expert scores as goals. Error scores were included despite not demonstrating CV, since speed should not been rewarded at the expense of error.

Another notable feature of this VR appendicectomy curriculum is its design for distributed rather than massed practice (favouring spacing intervals between practice sessions), as it is now well-documented that this results in superior learning and retention of skills [26]. Spacing intervals must be considered with respect to the importance of sleep and its possible role in the consolidation of learning (the memory consolidation hypothesis) and the potential detriment of continued practice during this process $[27,28]$. The optimal spacing interval is not yet known and may depend on task type [26], though so far there is evidence to suggest that daily or weekly sessions may be the most efficient for learning and retaining psychomotor skills [29].

A specified period of human instruction and feedback is also provided, although in reality this would likely be dictated by the logistical constraints of any simulation center that implements the curriculum and by the busy schedule of newly-qualified surgical trainees. A recent metaanalysis concluded that summary feedback (delivered infrequently and at the end of a session) was more effective than concurrent feedback (continuous feedback during each 
repetition), possibly due to cognitive overload in the latter, with the guidance hypothesis suggesting over-reliance and poorer performance upon its withdrawal [30]. The authors particularly recommend this educational approach to avoid the adoption of unsafe habits through actions that the simulators do not yet recognize. For example, crossing instruments or inserting scissors downwards towards the bowel and with open blades. Human feedback also promotes deconstruction of an automated task and conscious integration of better technique, which may at first prolong task completion time but may ultimately result in a higher quality of performance that goes unrecognized by the simulator (or indeed by this curriculum) [31].

Greater uncertainties exist surrounding the optimal session duration, the maximum number of sessions per day and the criteria for allowing participants to move on through the curriculum. So far only expert opinion has suggested that there should be no more than two training sessions per day [32,33], with each being limited to one hour $[33,34]$ to limit mental fatigue [35]. Many subscribe to this training regimen in their curricula, together with an accepted arbitrary criterion of having to demonstrate proficiency in two consecutive attempts before trainees are allowed to progress to the next stage $[8,36]$. Clearly, these curriculum design features require further study.

Previous authors have developed similar VR curricula for different procedures, such as laparoscopic cholecystectomy and salpingectomy, which have crucially demonstrated translation of VR performance to the real operating environment [14,37]. In one randomized and controlled transferability study, Ahlberg et al showed significant and consistent improvements in the performance of 10 consecutive laparoscopic cholecystectomies following VR training [14]. In another landmark randomized trial, Larsen et al demonstrated better and more rapid real salpingectomies in the VR procedural-trained group versus control [37].

Whilst this curriculum may ostensibly teach the psychomotor skills and the key steps in performing a laparoscopic appendicectomy, appendicitis is an acute inflammatory condition that arguably lacks the anatomical predictability of an elective cholecystectomy or an emergency salpingectomy. The intraoperative presentation of acute appendicitis varies in the 
position of the appendix and the degree of inflammation and adhesions. In reality, a surgeon must be able to perform a wide and unpredictable array of technical steps for each appendicectomy, in addition to the cognitive aspects of recognising the appendix base and judging the securest method of ligation. Therefore, not all components of the procedure are included in the present curriculum, and this may present a weakness. Furthermore, it does not address other important domains in the operating room, such as knowledge of anatomy and decision-making with task-switching in case of unexpected events [38], although it would be expected that this VR curriculum would complement these cognitive domains as part of the wider surgical training curriculum.

It is also recognized by the present authors that while 10 repetitions is greater than the number by which novices have been able to reach expert-level proficiency in previous studies of VR laparoscopy $[8,23,39]$, the shape of some of the presented learning curves suggests that ongoing practice may yield further improvements in novices' performance. This has been corroborated by others, with performance improvements and multiple plateaus seen towards the 30th repetition [40]. This would be especially important since some appendicectomy tasks in our curriculum feature some metrics where expert performance was not actually reached by the $10^{\text {th }}$ repetition. The current authors expect that further training, coupled with prior completion of the remainder of the curriculum, would see that expert proficiency is achievable, though this would need to be prospectively tested.

\section{CONCLUSIONS}

Many of the LAP Mentor basic psychomotor skills tasks and all component tasks of the laparoscopic appendicectomy module are able to distinguish novices from experts. Our analysis has informed the evidence-based construction of a novel VR curriculum for laparoscopic appendicectomy. Future study should prospectively assess the feasibility of its implementation and also its predictive validity, determining whether or not it leads to better performance in the real operating environment. 


\section{ACKNOWLEDGEMENT}

The authors thank Mr Zacharias Anastasiou for the statistical analysis support.

\section{REFERENCES}

1. Baird DLH, Simillis C, Kontovounisios C, Rasheed S, Tekkis PP. Acute appendicitis. BMJ 2017; 357: j1703

2. Findlay JM, Kafsi JE, Hammer C, Gilmour J, Gillies RS, Maynard ND. Nonoperative management of appendicitis in adults: a systematic review and meta-analysis of randomized controlled trials. J Am Coll Surg 2016; 223 (6): 814-824

3. Dai L, Shuai J. Laparoscopic versus open appendectomy in adults and children: a meta-analysis of randomized controlled trials. United European Gastroenterol J 2017; 5 (4): $542-553$

4. Figert PL, Park AE, Witzke DB, Schwartz RW. Transfer of training in acquiring laparoscopic skills. J Am Coll Surg 2001; 193 (5): 533-537

5. Deziel DJ, Millikan KW, Economou SG, Doolas A, Ko ST, Airan MC. Complications of laparoscopic cholecystectomy: A national survey of 4,292 hospitals and an analysis of 77,604 cases. Am J Surg 1993; 165 (1): 9-14

6. Flum DR, Koepsell T, Heagerty P, Pellegrini CA. The nationwide frequency of major adverse outcomes in antireflux surgery and the role of surgeon experience, 19921997. J Am Coll Surg 2002; 195 (5): 611-618

7. Larsen CR, Oestergaard J, Ottesen BS, Soerensen JL. The efficacy of virtual reality simulation training in laparoscopy: a systematic review of randomized trials. Acta Obstet Gynecol Scand 2012; 91 (9): 1015-28

8. Aggarwal R, Crochet P, Dias A, Misra A, Ziprin P, Darzi A. Development of a virtual reality training curriculum for laparoscopic cholecystectomy. Br J Surg 2009; 96 (9): 
1086-1093

9. Sinitsky DM, Fernando B, Berlingieri P. Establishing a curriculum for the acquisition of laparoscopic psychomotor skills in the virtual reality environment. Am J Surg 2012; 204 (3): 367-376

10. Maertens H, Vermassen F, Aggarwal R, Doyen B, Desender L, Van Herzeele I, Annemans L. Endovascular training using a simulation based curriculum is less expensive than training in the hybrid angiosuite. Eur J Vasc Endovasc Surg 2018; 56 (4): $583-590$

11. Wynn G, Lykoudis P, Berlingieri P. Development and implementation of a virtual reality laparoscopic colorectal training curriculum. Am J Sur 2018; 216 (3); 610-617

12. National Surgical Research Collaborative. Multicentre observational study of performance variation in provision and outcome of emergency appendicectomy. $\mathrm{Br} \mathrm{J}$ Surg 2013; 100 (9): 1240-1252

13. Yamaguchi S, Konishi K, Yasunaga T, Yoshida D, Kinjo N, Kobayashi K, leiri S, Okazaki K, Nakashima H, Tanoue K, Maehara Y, Hashizume M. Construct validity for eye-hand coordination skill on a virtual reality laparoscopic surgical simulator. Surg Endosc 2007; 21 (12): 2253-2257

14. Ahlberg G, Enochsson L, Gallagher AG, Hedman L, Hogman C, McClusky DA III, Ramel $\mathrm{S}$, Smith CD, Arvidsson D. Proficiency-based virtual reality training significantly reduces the error rate for residents during their first 10 laparoscopic cholecystectomies. Am J Surg 2007; 193 (6): 797-804

15. Aggarwal R, Grantcharov TP, Eriksen JR, Blirup D, Kristiansen VB, Funch-Jensen P, Darzi A. An evidence-based virtual reality training program for novice laparoscopic surgeons. Ann Surg 2006; 244 (2): 310-314

16. Wilson M, McGrath J, Vine S, Brewer J, Defriend D, Masters R. Psychomotor control in a virtual laparoscopic surgery training environment: gaze control parameters differentiate novices from experts. Surg Endosc 2010; 24 (10): 2458-2464

17. Palter VN, Graafland M, Schijven MP, Grantcharov TP. Designing a proficiency-based, 
content validated virtual reality curriculum for laparoscopic colorectal surgery: A Delphi approach. Surgery 2012; 151 (3): 391-397

18. Snyder CW, Vandromme MJ, Tyra SL, Hawn MT. Proficiency-based laparoscopic and endoscopic training with virtual reality simulators: a comparison of proctored and independent approaches. J Surg Educ 2009; 66 (4): 201-207

19. Zhang A, Hünerbein M, Dai Y, Schlag PM, Beller S. Construct validity testing of a laparoscopic surgery simulator (Lap Mentor ${ }^{\circledR}$ ). Evaluation of surgical skill with a virtual laparoscopic training simulator. Surg Endosc 2008; 22 (6): 1440-1444

20. McDougall EM, Corica FA, Boker JR, Sala LG, Stoliar G, Borin JF, Chu FT, Clayman RV. Construct validity testing of a laparoscopic surgical simulator. J Am Coll Surg 2006; 202 (5): 779-787

21. Woodrum DT, Andreatta PB, Yellamanchilli RK, Feryus L, Gauger PG, Minter RM. Construct validity of the LapSim laparoscopic surgical simulator. Am J Surg 2006; 191 (1): $28-32$

22. Brewin J, Nedas T, Challacombe B, Elhage O, Keisu J, Dasgupta P. Face, content and construct validation of the first virtual reality laparoscopic nephrectomy simulator. BJU Int. 2010; 106 (6): 850-854

23. Larsen CR, Grantcharov T, Aggarwal R, Tully A, Sørensen JL, Dalsgaard T, Ottesen B. Objective assessment of gynecologic laparoscopic skills using the LapSimGyn virtual reality simulator. Surg Endosc 2006; 20 (9): 1460-1466

24. Ericsson KA, Nandagopal K, Roring RW. Toward a science of exceptional achievement. Attaining superior performance through deliberate practice. Annals N Y Acad Sci 2009; 1172: 199-217.

25. Crochet P, Aggarwal R, Dubb SS, Ziprin P, Rajaretnam N, Grantcharov T, Eriksson KA, Darzi A. Deliberate practice on a virtual reality laparoscopic simulator enhances the quality of surgical technical skills. Ann Surg 2011; 253 (6): 1216-1222

26. Donovan JJ, Radosevich DJ. A meta-analytic review of the distribution of practice effect: now you see it, now you don't. J Appl Psychol 1999; 84 (5), 795-805 
27. Brashers-Krug T, Shadmehr R, Bizzi E. Consolidation in human motor memory. Nature 1996; 382 (6588): 252-255

28. Kuhn M, Wolf E, Maier JG, Mainberger F, Feige B, Schmid H, Burklin J, Maywald S, Mall V, Jung NH, Reis J, Spiegelhalder K, Kloppel S, Sterr A, Eckert A, Riemann D, Normann C, Nissen C. Sleep recalibrates homeostatic and associative synaptic plasticity in the human cortex. Nat Commun 2016; 7: 12455

29. De Win G, Van Bruwaene S, De Ridder D, Miserez M. The optimal frequency of endoscopic skill labs for training and skill retention on suturing: A randomized controlled trial. J Surg Educ. 2013; 70 (3): 384-393

30. Hatala R, Cook DA, Zendejas B, Hamstra SJ, Brydges R. Feedback for simulation-based procedural skills training: a meta-analysis and critical narrative synthesis. Adv Health Sci Educ Theory Pract 2014; 19 (2): 251-272

31. Boyle E, Al-Akash M, Gallagher AG, Traynor O, Hill ADK, Neary PC. Optimising surgical training: use of feedback to reduce errors during a simulated surgical procedure. Postgrad Med J 2011; 87 (1030): 524-528

32. Burden C, Oestergaard J, Larsen CR. Integration of laparoscopic virtual-reality simulation into gynaecology training. BJOG 2011; 118 (Suppl. 3): 5-10

33. Stefanidis D, Heniford BT. The formula for a successful laparoscopic skills curriculum. Arch Surg 2009; 144 (1): 77-82

34. Ericsson KA. Deliberate practice and the acquisition and maintenance of expert performance in medicine and related domains. Acad Med 2004; 79 (Suppl. 10): S70S81

35. Tsuda S, Scott D, Doyle J, Jones DB. Surgical skills training and simulation. Curr Probl Surg. 2009; 46 (4): 271-370

36. Strandbygaard J, Bjerrum F, Maagaard M, Winkel P, Larsen CR, Ringsted C, Gluud C, Grantcharov T, Ottesen B, Soerensen JL. Instructor feedback versus no instructor feedback on performance in a laparoscopic virtual reality simulator. A randomized trial. Ann Surg. 2013; 257 (5): 839-844 
37. Larsen CR, Soerensen JL, Grantcharov TP, Dalsgaard T, Schouenborg L, Ottosen C, Schroeder TV, Ottesen BS. Effect of virtual reality training on laparoscopic surgery: randomised controlled trial. BMJ 2009; 338: b1802

38. Bongers PJ, van Hove PD, Stassen LPS, Dankelman J, Schreuder HWR. A new virtualreality training module for laparoscopic surgical skills and equipment handling: can multitasking be trained? A randomized controlled trial. J Surg Educ 2015; 72 (2): 184191

39. Grantcharov TP, Bardram L, Funch-Jensen P, Rosenberg J. Learning curves and impact of previous operative experience on performance on a virtual reality simulator to test laparoscopic surgical skills. Am J Surg 2003; 185 (2): 146-149

40. Brunner WC, Korndorffer JR, Sierra R, Massarweh NN, Dunne JB, Yau CL, Scott DJ. Laparoscopic virtual reality training: are 30 repetitions enough? J Surg Res 2004; 122 (2): 150-156 


\section{FIGURE LEGENDS}

Figure 1: Screen captures of the laparoscopic appendicectomy five procedural tasks.

Color codes guide trainees throughout the surgical steps. Task 1: Dissecting the mesenteric window; Task 2: Dissecting the mesoappendix and clipping the artery; Task 3: Clipping the artery and ligating the appendix using a ligating loop; Task 4: Division of the mesoappendix and base of the appendix using a stapler; Task 5: Control of the artery using energy.

\section{Figure 2. Total time learning curves for appendicectomy tasks LAPT1-5 and LAFP.}

Median and error bars representing 95\% confidence intervals are presented for novices' repetitions 1 through 10 (exception of LAPT5, displaying mean and standard deviation due to data normality). Both construct validity and learning curves were highly significant for all of these metrics $(p \leq 0.002)$.

\section{Figure 3. Curriculum for laparoscopic appendicectomy on the LAP Mentor.}

The median of experts' scores from the construct validity assessment were set as the proficiency criteria for progression through the curriculum. The median scores have similarly been used elsewhere $[9,15,16,18-19]$, balancing the requirements of an effective and achievable goal. Participants following the curriculum may practice for up to 45 minutes per session, with no more than one session per day. Progression occurs when criteria have been met only at the start of the session to ensure consistency and retention of skill from the previous session, and to guarantee a greater number of spaced sessions. Those unable to progress after five sessions must request an instructor feedback session. 